\title{
Multi-user Detection Based on Weight approaching particle filter in Impulsive Noise
}

\author{
XIAN Jin long ${ }^{1,}$ a , LI Sheng Jie ${ }^{2, b}$ \\ ${ }^{1}$ College of Information Science and Engineering, Henan University of \\ Technology, Zhengzhou, 450001, Henan, P.R. China. \\ ${ }^{2}$ College of Information Science and Engineering, Henan University of \\ Technology, Zhengzhou, 450001, Henan, P.R. China. \\ a13991339876@vip.sina.com, ${ }^{b}$ lishengjie0318@163.com
}

\begin{abstract}
.
To solve the problem of particle degeneracy and sample impoverishment in conventional particle filter, we propose the weight approaching particle filter(WAPF) to increase the particle diversity before resampling step for adaptive multi-user detection (MUD) in synchronous code division multiple access (CDMA) system.. In the resampling step, particles are classified into two groups according to their particle-weights, and then the particles with the smaller weights are replaced by the mean of the two group particles, so that the particles can approach from the low likelihood region to the high likelihood region. Similar to the carrier wavemethod, the chaotic perturbation resampling method adopts the chaotic variable with the property of global ergodicity to ameliorate the diversity of samples and reduce the computation load. Simulation results demonstrate the feasibility of the improved particle filter.
\end{abstract}

Keywords: Particle filter, Multi-user detection, weight approaching, Non-Gaussian noise

\section{Introduction}

Multi-user detection technology is a good solution to effectively resist multiple access interference (MAI) and distance effect in synchronous code division multiple access (CDMA) system[1]. In 1993, N.J.Gordon recommended to use particle filter (PF) method to track the signal in the literature [2]. This technique can be applied to any nonlinear and non-Gaussian systems that can be 
represented by a state-space model. This feature makes the algorithm has strong adaptability.

A lot of noise in actual life are non-Gaussian noise[3]. Particle filter algorithm does not require the noise model is Gaussian noise. So PF algorithm can be applied in the field of multi-user detection, can effectively reduce the bit error rate and has applicable value.

This paper is organized as follow: Section 2 includes the model of the CDMA system and the presentation of two kinds of non-Gaussian noises; the application of standard particle filter algorithm for multi-user detection is presented in Section 3; the application of importance of weight approaching particle filter algorithm for multi-user detection is presented in Section 4; Simulation results and conclusions are described in Section 5.

\section{CDMA system model}

Consider a synchronous CDMA system with $\mathrm{K}$ users. The $\mathrm{k}$-th receive signal is [1][4]:

$$
\mathrm{r}(t)=\sum_{k=1}^{K} A_{k}(t) g_{k}(t) b_{k}(t)+n(t)
$$

In the formula, $A_{K}$ means the amplitude of the k-th signal; $\mathrm{g}_{k}$ means spread spectrum waveform of the $\mathrm{k}$-th signal; $\mathrm{b}_{k}$ means the $\mathrm{k}$-th user data, the value is $\pm 1 ; n(t)$ is background noise.

Judgment performed by a matched filter, $y_{k}$ for the K-th matched filter output, expressed as:

$$
\rho_{i, k}=\frac{1}{T_{b}} \int_{0}^{T_{b}} g_{k}(t) g_{i}(t) d t
$$

Can be obtained by expanding:

$$
\begin{aligned}
& y_{k}=\int_{0}^{T} r(t) g_{k}(t) d t=\int_{0}^{T}\left(\sum_{k=1}^{K} A_{k}(t) g_{k}(t) b_{k}(t)+n(t)\right) g_{k}(t) d t \\
= & A_{k} b_{k}+M A I_{k}+z_{k}
\end{aligned}
$$

$A_{k} b_{k}$ means the signal of the k-th user; $z_{k}$ is noise; $M A I_{k}$ is multiple access interference (MAI) which is generated by other users. 
In order to facilitate processing and analysis, the received vector can be expressed as matrix.

$$
y=R A b+z
$$

Where $A=\operatorname{diag}\left\{A_{1}, A_{2}, \cdots, A_{k}\right\}$ is a diagonal matrix of received signal amplitude. $b=\left[b_{1}, b_{2}, \cdots, b_{k}\right]$ is user data, $\mathrm{R}$ is symmetric correlation matrix of $K \times K \operatorname{order}\left(\rho_{i, k}=\rho_{k, i}\right) \cdot z=\left[z_{1}, z_{2}, \cdots, z_{k}\right]^{T}$.

Colicky factorization can be used to R. such that $R=F^{T} F$. So we obtain:

$\bar{y}=F^{-T} y=F^{-T} F A b+F^{-T} z=F A b+\bar{z}$

$\bar{y}$ is called the whitened matched filter output. Expression of the received signal is as follows:

$$
\overline{y_{k}}=\sum_{l=1}^{K} F_{k, l} a_{l} b_{l}+\overline{z_{k}}
$$

On the basis of the spatial model, the purpose of multi-user detection is to detect signals of the users $b_{1: k}=\left\{b_{1}, b_{2}, \cdots, b_{k}\right\}$ from the matched filter output signals $\bar{y}_{1: k}=\left\{\bar{y}_{1}, \bar{y}_{2}, \cdots, \bar{y}_{k}\right\}$.

\section{Non-Gaussian noise simulation}

In practice, there are some kinds of noise that they are not happen often, but with a strong impact. These kinds of noise are not Gaussian noise, such as thunder and lightning, all kinds of machine motors, etc. do not have Gaussian nature. The following briefly discusses two models of the non-Gaussian noise.

A. Laplace noise

Laplace probability density function (PDF) has an obvious smearing. This is difference between the Laplace PDF and the Gaussian PDF. Laplace probability density function[3]:

$$
p(x)=\frac{1}{\sqrt{2 \sigma^{2}}} \exp \left(-\sqrt{\frac{2}{\sigma^{2}}}|x|\right),-\infty<x<+\infty
$$


In the formula, $\sigma^{2}$ parameter is variance or power of noise.

B. Alpha noise

If $X$ is subject to the Alpha stable distribution, its characteristic function is:

$$
\left.\phi(u)=\exp j a u-\gamma u^{\alpha}[1+j \beta \operatorname{sgn}(u) \omega(u, \alpha)]\right\}
$$

$$
\omega(u, \alpha)=\left\{\begin{array}{l}
\tan (\pi \alpha / 2), \cdots, \alpha \neq 1 \\
(2 / \pi) \log |u|, \cdots, \alpha=1
\end{array}\right.
$$

$$
\operatorname{sgn}(u)=\left\{\begin{array}{l}
1, \cdots \cdot u>0 \\
0, \cdots \cdot u=0 \\
-1, \cdots u<0
\end{array}\right.
$$

In the formula, $\alpha \in(0,2]$ is characteristic index which determines the degree of the distribution pulse characteristics. When $\alpha=2$, it is Gaussian distribution. When $0<\alpha<2$, this distribution is called fractional lower order Alpha stable distribution. $-1<\beta<1$ is called symmetry parameter which can control the gradient of the distribution. When $\beta=0$, it is a symmetric $\alpha$-stable distribution, referred to as $S \alpha S . \gamma$ is called scattering coefficients which can control the dispersion measure about the samples relative to the mean. The noise power can be expressed approximately as $2 \gamma$, but $2 \gamma$ is not equal completely to the true noise power. Signal to Noise Ratio (SNR) can be expresses as $S N R=S / 2 \gamma(S$ is the signal power $)$.

\section{Multi-user detection based on standard particle filter algorithm}

The particle filter is a Monte Carlo method based on Bayesian theory. Its core idea is that using the samples and their corresponding weights to express the posterior probability density function then we can use the posterior probability density to obtain the estimated value of the state. 
The main steps of multi-user detection based on particle filter are as follows [5]:

Step 1: Sampling for the k-th user, making $x_{k}^{i}: q\left(x_{k} \mid x_{1: k-1}^{i}, \bar{y}_{1: k}\right)$.

Step 2: calculate the weights of the particles. $\omega_{k}^{i} \propto=\omega_{k-1}^{i} \frac{p\left(\bar{y}_{k} \mid x_{k}^{i}\right) p\left(x_{k}^{i} \mid x_{k-1}^{i}\right)}{q\left(x_{k}^{i} \mid x_{1: k-1}^{i}, \bar{y}_{1: k}\right)}$

Step 3: Normalizing the weights . $\omega_{k}^{i}=\omega_{k}^{i} / \sum_{i=1}^{N_{s}} \omega_{k}^{i}$

Step 4: Resampling for the particles.

Step 5: According to the Maximum A Posterior rule to estimate the signals of the k-th user.

Step 6: Turn to step1, and estimating the signals of the next user.

\section{Multi-user detection based on weight approaching particle filter}

Particle degradation is a major problem of conventional particle filter. Resampling can solve problems of particle degradation to some extent, but it also brings a particle depletion problem[3]. The so-called particle depletion is that those particles having a larger weight is selected times, lower weight particles gradually disappear after resampling. It makes that particles loss the diversity and are not sufficient to description posterior probability. To solve these problems, this paper presents a new particle filter improved method that weight approaching particle filter algorithm (WAPF). By preprocessing the prediction set of particles and increasing effective number of particles well improve the performance of conventional particle filter.

The basic idea of weight approaching particle filter algorithm is that: After each sampling, the weight of the particles are sorted in ascending order; A number of high-weight particles that are selected referring to the effective sample of fissile breeding start to do fissile breeding. The method of fissile breeding is starting from the smallest particle weight, finding the weighted average between it and high weight particle, using the weighted average regeneration particle to cover original low weight particles in turn, to regenerate particles as sampling particles recalculating the weight of particle. So the 
particles can approach from the low likelihood region to the high likelihood region.

If resampling conditions are met, the next step is chaotic perturbation resampling (CPR). Its main idea is to introduce variability thinking in evolutionary algorithms. The maximum weight of particles is going to be chaotic mutation and the number of variation of particles is the number of degraded particles. Then the weights of variation of particles were calculated, variation of particles are used to cover degraded particles for state estimation. CPR's process is summarized as follows:

$$
\begin{aligned}
& \text { IF } N_{\text {eff }}<\text { threshold } \\
& \quad x_{k}^{i} \text { are sorted by its weight } \\
& \quad \text { FOR } j=1: N-N_{\text {eff }} \\
& \quad x_{k}^{i}=x_{k}^{*}+\operatorname{chaos}(Q) \\
& x_{k}^{*} \text { is the largest weight particle, } \operatorname{chao}(Q) \text { mean chaotic }
\end{aligned}
$$

perturbation, meanwhile require all new weights $\omega_{k}^{j}=1 / N$, Q is chaotic variable.

\section{END}

END

In summary, the steps of multi-user detection based on auxiliary particle filter are as follows:

Step 1: Sampling for the k-th user, making $x_{k}^{i}: q\left(x_{k} \mid x_{1: k-1}^{i}, \bar{y}_{1: k}\right)$.

Step 2: According to equation (11) to calculate the weights of the particles.

Step 3: Normalizing the weights according to equation (12).

Step 4: Fissile breeding according to the above.

Step 5: Recalculated weights. If $\quad N_{\text {eff }}<$ threshold, CPR for the particles.

Step 6: According to the Maximum A Posterior rule to estimate the signals of the k-th user.

Step 7: Turn to step1, and estimating the signals of the next user.

\section{Simulation results and conclusions}

Consider a synchronous CDMA system, and we select 5 users, 40000 information bits, 31-bit gold spread-spectrum code and the user power partial value is 10. Channel noises are additive Gaussian noise, Laplace noise and Alpha stable noise. The range of signal to noise ratio (SNR) for all users is $-4 \sim 10 \mathrm{~dB}$. The number of particles is 200 . 
Figure1: We can find that the performance of the WAPF algorithm is better than the PF algorithm in Gaussian noise. WAPF algorithm can improve the performance of the system.

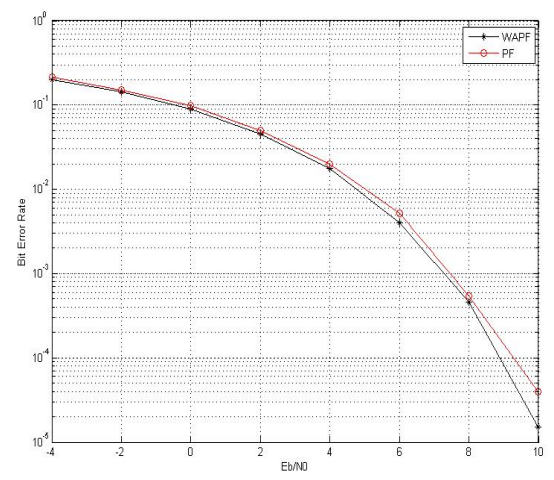

Figure1. The BER of PF detection and WAPF detection.

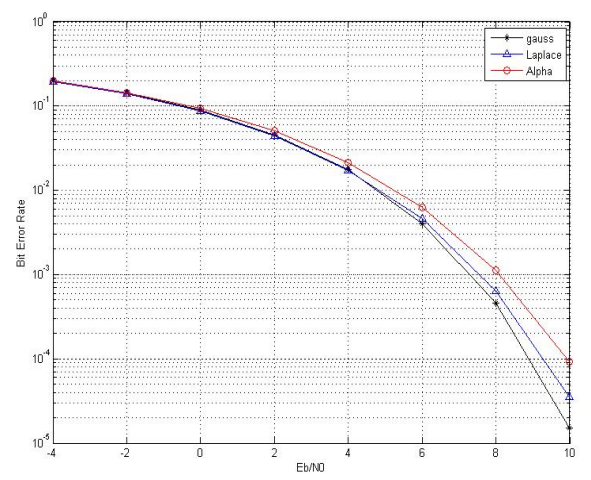

Figure2. BER of WAPF detection under three kinds of noise

Figure2: This figure analyzes the error code performance of WAPF detection aiming to Gaussian noise, Alpha stable noise and Laplace noise. We can see from the Figure 2 that the error code performance of the Gaussian noises and the Laplace noise are almost same. The result also proves that the applicability of the WAPF algorithm is well in the non-Gaussian system. Therefore, this algorithm has practical reference value. 


\section{Acknowledgment}

Sincerely thank the Scientific Research Fund of Henan University of Technology.

\section{References}

[1] Dou zhongzhao, Lei xiang, "CDMA Wireless Communications Theory", Tsinghua University Press, China, 2004.

[2] N.J.Gordan, D.J.Salmond, A.F.M.Smith, "Novel approach to non-linear and non-Gaussian Bayesian state estimation", IEEE Proc.F, vol.140, no.2, pp.107-113, 1993.

[3] Steven M.Kay Edited, translated by Peng-fei Luo, "Statistics based on signal processing: estimation and detection theory", Electronic Industry Press, China, 2006.

[4] Xian Jinlong, Liu Zhi, Zhang Qinghui, "The Application of Accelerated ECM Algorithm to MUD", International Journal of Digital Content Technology and its Applications, vol.6, no.2, pp.145-152, 2012.

[5] Zhimin CHEN, Yuming BO, Panlong WU, Mingfeng YIN. "Quasi-Monte Carlo Particle filter Fault Prognosis Algorithm Based on GRNN", JCIT, vol.7, no.7, pp.96-103, 2012. 